\title{
Dominant polar surfaces of colloidal II-VI semiconductor nanocrystals enabled by cation exchange
}

Aixiang Wang* ${ }^{\dagger}$, Wenjie Wang ${ }^{\dagger}$, Jiayi Chen ${ }^{\ddagger}$, Rundong Mao ${ }^{\ddagger}$, Yingping Pang Yunguo Li* ${ }^{\S}$, Wei Chen ${ }^{\ddagger}$, Dechao Chen ${ }^{\ddagger}$, Derek $\mathrm{Hao}^{\perp}$, Bing-Jie $\mathrm{Ni}^{\perp}$, Martin Saunders"l, Guohua Jia**

'School of Chemistry and Chemical Engineering, Linyi University, Linyi 276005, P.R. China.

${ }^{\ddagger}$ Curtin Institute of Functional Molecules and Interfaces, School of Molecular and Life Sciences, Curtin University, Bentley, WA 6102, Australia

${ }^{\S}$ Department of Earth Sciences, Faculty of Mathematical and Physical Sciences, University College London, Gower Street, London WC1E 6BT, UK.

${ }^{\perp}$ Centre for Technology in Water and Wastewater (CTWW), School of Civil and Environmental Engineering, University of Technology Sydney (UTS), Ultimo, NSW 2007, Australia

"Centre for Microscopy, Characterization and Analysis (CMCA), The University of Western Australia, Clawley, WA 6009, Australia

*Corresponding author. Email: guohua.jia@curtin.edu.au (G.J); wangaixiang1974@163.com (A.W.); yunguo.li@ucl.ac.uk (Y.L.) 


\section{Experimental Procedures}

Chemicals: Copper(I) thiocyanate [CuSCN] (99\%), copper (I) chloride (97\%), $\mathrm{Zn}\left(\mathrm{NO}_{3}\right)_{2} \cdot 6 \mathrm{H}_{2} \mathrm{O}(99 \%), \mathrm{ZnCl}_{2}(99.995 \%)$, zinc diethyldithiocarbamate (97\%), ethylene glycol (EG) (99.8\%), tetramethylammonium hydroxide pentahydrate $(\geq 97 \%)$, sulphur powder $(99.999 \%)$, octadecene (ODE) (97\%), oleylamine (approximate $\mathrm{C}_{18}$ content $70 \%$ ), tetramethylammonium hydroxide solution (25 wt. \% in $\left.\mathrm{H}_{2} \mathrm{O}\right)$, chloroform $(99 \%$ anhydrous), methanol (99.8\% anhydrous), $5 \mathrm{w} \%$ Nafion solution, ethyl alcohol (anhydrous, $\geqslant 99.5 \%)$ and $\mathrm{HCl}(37 \%)$ were purchased from Sigma-Aldrich. All chemicals were used as received without further purification.

Synthesis of $\mathrm{Cu}_{2-\mathrm{x}} \mathrm{S}$ hexagonal nanoplates: $\mathrm{Cu}_{2-\mathrm{x}} \mathrm{S}$ hexagonal nanoplates were synthesized according to a reported approach. ${ }^{1}$ In a typical synthesis, $0.1 \mathrm{mmol}$ of copper(I) thiocyanate $[\mathrm{CuSCN}]$ was mixed with $7 \mathrm{~mL}$ of oleylamine and $8 \mathrm{~mL}$ of ODE in a $50 \mathrm{~mL}$ three neck round bottom flask. The mixture was degassed at $110{ }^{\circ} \mathrm{C}$ for $30 \mathrm{~min}$. Then the temperature was raised to $280{ }^{\circ} \mathrm{C}$ under $\mathrm{N}_{2}$ flow, and kept at this temperature for another $5 \mathrm{~min}$. The temperature was cooled down to room temperature and a $15 \mathrm{~mL}$ mixture of toluene/methanol $=1: 1$ was added. The whole resulting product was centrifuged and washed three times with a $15 \mathrm{~mL}$ mixture of toluene/methanol $=1: 1$. The products were naturally dried in the glovebox.

Synthesis of ultralarge lateral sized $\mathrm{Cu}_{2-x}$ S hexagonal nanoplates: Ultralarge lateral sized $\mathrm{Cu}_{2-\mathrm{x}} \mathrm{S}$ hexagonal nanoplates were synthesized according to a reported approach. ${ }^{2}$ Purified $\mathrm{Cu}_{2-\mathrm{x}} \mathrm{S}$ hexagonal nanoplates from $5 \mathrm{~mL}$ crude solution were dissolved in $5 \mathrm{~mL}$ oleylamine and the mixture was degassed at room temperature and refilled with $\mathrm{N}_{2}$ for 
three times. Then the mixture was heated up to $110{ }^{\circ} \mathrm{C}$ and kept at this temperature for 30 minutes under vacuum. Then the solution was heated up to $230{ }^{\circ} \mathrm{C}$ and aliquots were taken from time to time in order to monitor the evolution of $\mathrm{Cu}_{2-\mathrm{x}} \mathrm{S}$ hexagonal nanoplates. After the reaction proceeded at $230{ }^{\circ} \mathrm{C}$ for a desired time, the heating mantle was removed and the solution was allowed to cool to room temperature. Purification of $\mathrm{Cu}_{2-\mathrm{x}} \mathrm{S}$ hexagonal nanoplates with large lateral size is the same as described above.

Synthesis of wurtzite ZnS dots: Wurtzite $\mathrm{ZnS}$ dots were synthesized according to a reported approach. ${ }^{3}$ Sulfur precursor stock solution was prepared by dissolving $5 \mathrm{mmol}$ of thiourea in $30 \mathrm{~mL}$ of EG. In a typical synthesis, $5 \mathrm{mmol}$ of anhydrous $\mathrm{ZnCl}_{2}$ and 10 mmol of tetramethylammonium hydroxide were dissolved into $30 \mathrm{~mL}$ of EG and heated to $100{ }^{\circ} \mathrm{C}$ in a three-neck round bottom flask. Under vigorous magnetic stirring, sulfur precursor stock solution was then quickly injected into the flask. The mixed solution was clear until the solution was heated to $150{ }^{\circ} \mathrm{C}$; then after about 10 min the solution becaes milky white, indicating the formation of $\mathrm{ZnS}$ nanocrystals. Then the mixture was heated to $150-160{ }^{\circ} \mathrm{C}$. After maintaining the reaction solution at this temperature for $2 \mathrm{~h}$, the color of reaction solution became milky white mixed with light yellow. The solution was heated further to $194{ }^{\circ} \mathrm{C}$ and refluxing for another $1 \mathrm{~h}$ before cooling down to room temperature.

Synthesis of wurtzite ZnS nanowires. ZnS nanowire were synthesized according to a previously reported approach with some modifications. ${ }^{4}$ In a typical synthesis, $0.05 \mathrm{mmol}$ $(18 \mathrm{mg})$ of zinc diethyldithiocarbamate and $10 \mathrm{~mL}$ of oleylamine were mixed in a threeneck flask. The mixture was degassed and refilled with Ar three times at room 
temperature and then heated to $110^{\circ} \mathrm{C}$ and kept at this temperature for $0.5 \mathrm{~h}$. Then the temperature was raised from 110 to $230{ }^{\circ} \mathrm{C}$ in $6 \mathrm{~min}$. After $20 \mathrm{~min}$ at $230{ }^{\circ} \mathrm{C}$, the reaction was quenched by removing the heating mantle.

Synthesis of wurtzite ZnS nanorods: Wurtzite $\mathrm{ZnS}$ nanorods were synthesized according to a reported approach. ${ }^{5}$ Purified $\mathrm{ZnS}$ nanowires were redissolved in oleylamine in a three-neck flask. The reaction mixture was degassed and refilled with Ar for three times at room temperature and then heated to $110^{\circ} \mathrm{C}$ and kept at this temperature for $10 \mathrm{~min}$. Then the reaction solution was heated to $280{ }^{\circ} \mathrm{C}$ in $10 \mathrm{~min}$ and kept at this temperature for 5-60 min. Aliquots were taken from time to time. The reaction was terminated by removing the heating mantle.

Synthesis of wurtzite $\mathrm{ZnS}$ rectangular nanoplatelets: Wurtzite $\mathrm{ZnS}$ rectangular nanoplatelets were synthesized according to a reported approach. ${ }^{6}$ Monodisperse $\mathrm{ZnS}$ rectangular nanoplatelets were synthesized using a one-pot synthetic method. In a typical synthesis, $\mathrm{Zn}\left(\mathrm{NO}_{3}\right)_{2} \cdot 6 \mathrm{H}_{2} \mathrm{O}(0.3 \mathrm{mmol}, 89 \mathrm{mg})$ and sulfur $(0.9 \mathrm{mmol}, 28.9 \mathrm{mg})$ were mixed with $5 \mathrm{~mL}$ octylamine and $10 \mathrm{~mL}$ oleylamine in a $50 \mathrm{~mL}$ three-neck flask. The mixture was heated to $110^{\circ} \mathrm{C}$ and kept at this temperature for $30 \mathrm{~min}$. The temperature was then held at $170{ }^{\circ} \mathrm{C}$ for $6 \mathrm{~h}$, before removal of the heating mantel and cooled down to room temperature. The product solution was diluted in chloroform, and the rectangular nanoplatelets were precipitated by adding methanol with the aid of centrifugation. Synthesis of wurtzite ZnS hexagonal nanoplates with dominant $\{002\}$ polar surfaces: ZnS hexagonal nanoplates were prepared by exchanging $\mathrm{Cu}^{+}$with $\mathrm{Zn}^{2+}$ in $\mathrm{Cu}_{2-\mathrm{x}} \mathrm{S}$ nanocrystals using cation exchange reactions. $0.1 \mathrm{M}$ zinc oleate stock solution was prepared by 
dissolving $\mathrm{Zn}\left(\mathrm{NO}_{3}\right)_{2} \cdot 6 \mathrm{H}_{2} \mathrm{O}(473.4 \mathrm{mg}, 2.5 \mathrm{mmol})$ into a mixture of oleic acid $(6.94 \mathrm{~mL})$ and ODE $(18 \mathrm{~mL})$ at $220{ }^{\circ} \mathrm{C}$. Cleaned $5 \mathrm{mg} \mathrm{Cu} \mathrm{Cu}_{1.75} \mathrm{~S}$ hexagonal nanoplates were dissolved into anhydrous chloroform and then the solution was injected into $5 \mathrm{~mL}$ oleylamine solution in a three neck flask. The mixture was degassed at $110{ }^{\circ} \mathrm{C}$ for 10 minutes, and then it was heated up to $220{ }^{\circ} \mathrm{C}$. When the temperature was stabilized, $1 \mathrm{~mL}$ zinc oleate stock solution $(0.1 \mathrm{M})$ was swiftly injected into the flask while stirring. The solution in the flask was gradually changed from brown to colorless. After 25 minutes at this temperature the heating mantle was removed and the mixture was cooled down to room temperature.

Synthesis of $\mathrm{Cu}_{2} \mathrm{~S}-\mathrm{ZnS}$ hetero-nanoplates through partial cation exchange: $\mathrm{Cu}_{2} \mathrm{~S}-\mathrm{ZnS}$ hetero-nanoplates were prepared by exchanging $\mathrm{Cu}^{+}$with $\mathrm{Zn}^{2+}$ in $\mathrm{Cu}_{2} \mathrm{~S}$ nanocrystals using partial cation exchange reactions. $0.02 \mathrm{M}$ zinc-oleylamine solution was prepared by dissolving $\mathrm{ZnCl}_{2} \cdot 6 \mathrm{H}_{2} \mathrm{O}(27.3 \mathrm{mg}, 0.2 \mathrm{mmol})$ into a mixture of oleylamine $(6 \mathrm{~mL})$ and ODE (4 mL) in a three neck flask and degassed for 30 minutes at $120^{\circ} \mathrm{C}$. Cleaned 44.3 mg $\mathrm{Cu}_{1.94} \mathrm{~S}$ hexagonal nanoplates were dissolved into $3 \mathrm{~mL}$ oleylamine and then the solution was injected into the zinc-oleylamine solution in a three neck flask at $150{ }^{\circ} \mathrm{C}$, and then $2 \mathrm{~mL}$ trioctylphosphine was injected at $180^{\circ} \mathrm{C}$. Then the mixture was heated up to $250{ }^{\circ} \mathrm{C}$. The solution in the flask was gradually changed from dark brown to light brown. After 4 minutes at this temperature, the heating mantle was removed and the mixture was cooled down to room temperature.

Sample Characterization: UV-vis absorption spectroscopy was performed on a Perkin Elmer Lambda UV/VIS/NIR Spectrometer. Powder X-ray diffraction (XRD) patterns 
were obtained using $\mathrm{Cu} \mathrm{K} \alpha(\lambda=1.5406 \AA)$ photons from an X'per PRO (PANalytical) Xray diffractometer operated at $40 \mathrm{kV}$ and $40 \mathrm{~mA}$. Transmission electron microscopy (TEM) was performed using a JEOL 2100 transmission electron microscope. Highresolution TEM (HRTEM), high angle annular dark field-scanning transmission electron microscopy (HAADF-STEM) and STEM-energy-dispersive X-ray spectroscopy (STEMEDX) were performed on an FEI Titan G2 80-200 high-resolution transmission electron microscope.

Electrochemical measurements: Electrochemical measurements were carried out on an electrochemical working station (CHI-760E). The saturated calomel electrode was used as the reference electrode. All potentials were referred to reversible hydrogen electrode by adding a value of $(0.242+0.059 \mathrm{pH}) \mathrm{V}$. A carbon rod and glassy carbon electrode were used as the counter and working electrode. Catalyst ink was prepared by dispersing catalyst power in $1 \mathrm{~mL}$ ethanol containing $50 \mu \mathrm{L}$ Nafion solution (5 wt\%) under sonification. $5 \mu \mathrm{L}$ of the above solution was drop-cast on a mirror-finished glassy carbon electrode. Electrolyte was $1 \mathrm{M} \mathrm{HCl}$ solution.

DFT calculation details: Density functional theory (DFT) calculations were performed using the Vienna $A b$ initio Simulations Package (VASP) ${ }^{7,8}$ and projected augmented wave (PAW) ${ }^{9-11}$ method (with $2 s^{2} 2 p^{4}, 3 s^{2} 3 p^{4}, 3 d^{10} 4 s^{2}$ as valence electrons for $\mathrm{O}, \mathrm{S}$ and $\mathrm{Zn}$, respectively). The exchange-correlation interaction was treated with generalized gradient approximation (GGA) in the Perdew, Burke and Ernzerhof (PBE) ${ }^{12}$ parameterization. For strongly localized $d$ electrons, GGA has systematic and noncancelling errors. ${ }^{13}$ Therefore, we adopted GGA+U $(\mathrm{U}=8 \text { and } \mathrm{J}=1)^{14,15}$ to correct the 
self-interaction and overdelocalized $d$ states. The cutoff energy of plane-wave basis was set to $450 \mathrm{eV}$, and integrations over the first Brillouin zone were calculated using a Gamma-centred k-point set of $6 \times 6 \times 4$.

With these settings, the total energy was able to converge within $1 \mathrm{meV} / \mathrm{atom}$. The wurtzite $\mathrm{ZnS}$ (space group $P 6_{3} m c$, No. 186) structure was fully relaxed using GGA+U, and the energy was converged within $10^{-6} \mathrm{eV} / \mathrm{cell}$ and the force was converged to less than $10^{-4} \mathrm{eV} / \AA$. A vacuum spacing no less than $15 \AA$ was used in all slab calculations with the same cutoff energy and similar k-point density to bulk calculations.

\section{Percentages of polar surfaces of nanocrystals with different morphologies}

Spherical dots: Assume the diameter of a spherical dot illustrated in Scheme 1 in the main article is $4 \mathrm{~nm}$, its projection is a hexagon with the diagonal of being $4 \mathrm{~nm}$.

Area of the side surfaces of a truncated dot: $(2 \times 2 \times 6)=24 \mathrm{~nm}^{2}$;

Area of the end surfaces of a truncated dot: $0.5 \times 1 \times(1.732 / 2) \times 6 \times 2=5.20 \mathrm{~nm}^{2}$;

Area of the connecting (truncated) surfaces a truncated dot: $0.5 \times(1+2) \times(1.732 / 2) \times 12=15.59 \mathrm{~nm}^{2} ;$

Percentages of polar facets $=5.2 /(5.2+15.59+24)=11.6 \%$.

Nanorods: Assume the diameter of nanorods illustrated in Scheme 1 in the main article is $4 \mathrm{~nm}$ and the length is $20 \mathrm{~nm}$, the polar surfaces and truncated surfaces are similar to those of spherical dots discussed above, the only difference is the side facets, which are elongated.

Area of the side surfaces of a nanorod: $(2 \times 20 \times 6)=240 \mathrm{~nm}^{2}$; 
Area of the end surfaces of a nanorod: $0.5 \times 1 \times(1.732 / 2) \times 6 \times 2=5.20 \mathrm{~nm}^{2}$;

Area of the connecting (truncated) surfaces a truncated dot: $0.5 \times(1+2) \times(1.732 / 2) \times 12=15.59 \mathrm{~nm}^{2} ;$

Percentages of polar facets $=5.2 /(5.2+15.59+240)=2.0 \%$.

Rectangular nanoplatelets: Assume the width of the rectangular nanoplatelet illustrated in Scheme 1 in the main article is $13 \mathrm{~nm}$, the length is $60 \mathrm{~nm}$ and the thickness is $1.4 \mathrm{~nm}$, the non-polar $\{110\}$ surfaces became the dominant surfaces.

Area of the polar facets $=1.4 \times 13 \times 2=36.4 \mathrm{~nm}^{2}$;

Area of side facets (100): $1.4 \times 60 \times 2=168 \mathrm{~nm}^{2}$;

Area of side facets (110): $13 \times 60 \times 2=1560 \mathrm{~nm}^{2}$;

Percentages of polar facets $=36.4 /(168+36.4+1560)=2.1 \%$.

Hexagonal nanoplates: Assume the lateral size of a hexagonal nanoplate illustrated in Scheme 1 in the main article is $500 \mathrm{~nm}$ and the thickness is $5 \mathrm{~nm}$, the polar $\{002\}$ surfaces became the dominant surfaces.

Area of the dominant polar $\{002\}$ surfaces of a hexagonal nanoplate: $0.5 \times(500+1000) \times 500 \times(1.732 / 2) \times 2=649500 \mathrm{~nm}^{2} ;$

Area of the side facets of a hexagonal nanoplate: $(5 \times 500 \times 6)=15000 \mathrm{~nm}^{2}$;

Percentages of polar surfaces $=649500 /(649500+15000)=649500 /(664500)=97.8 \%$. 


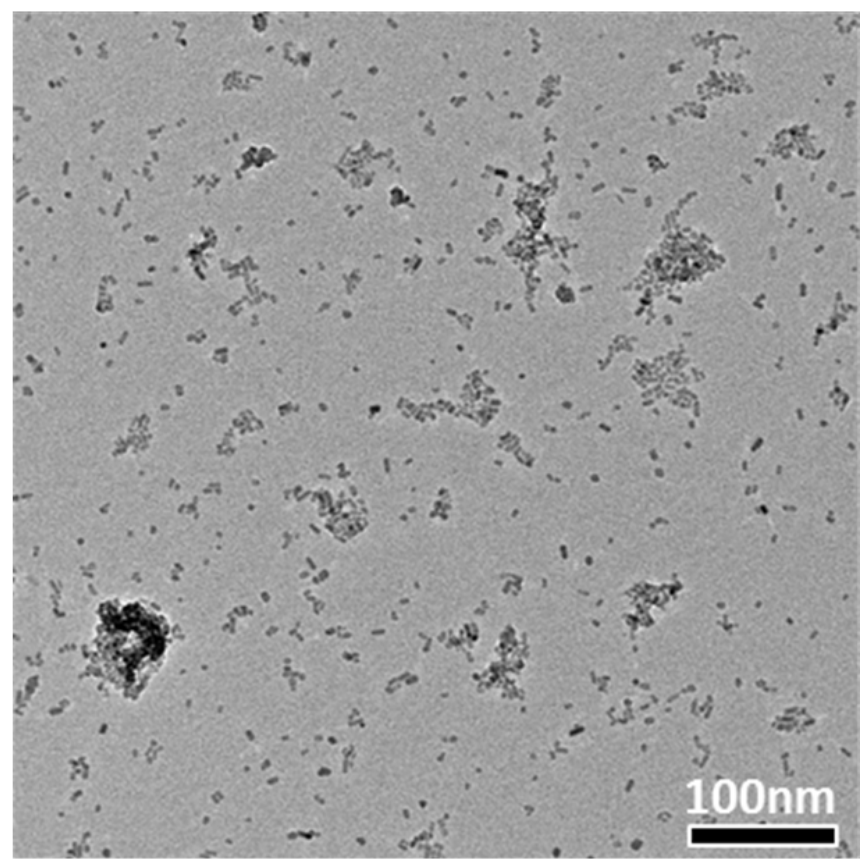

Figure S1. TEM image of wurtzite $\mathrm{ZnS}$ dots. 


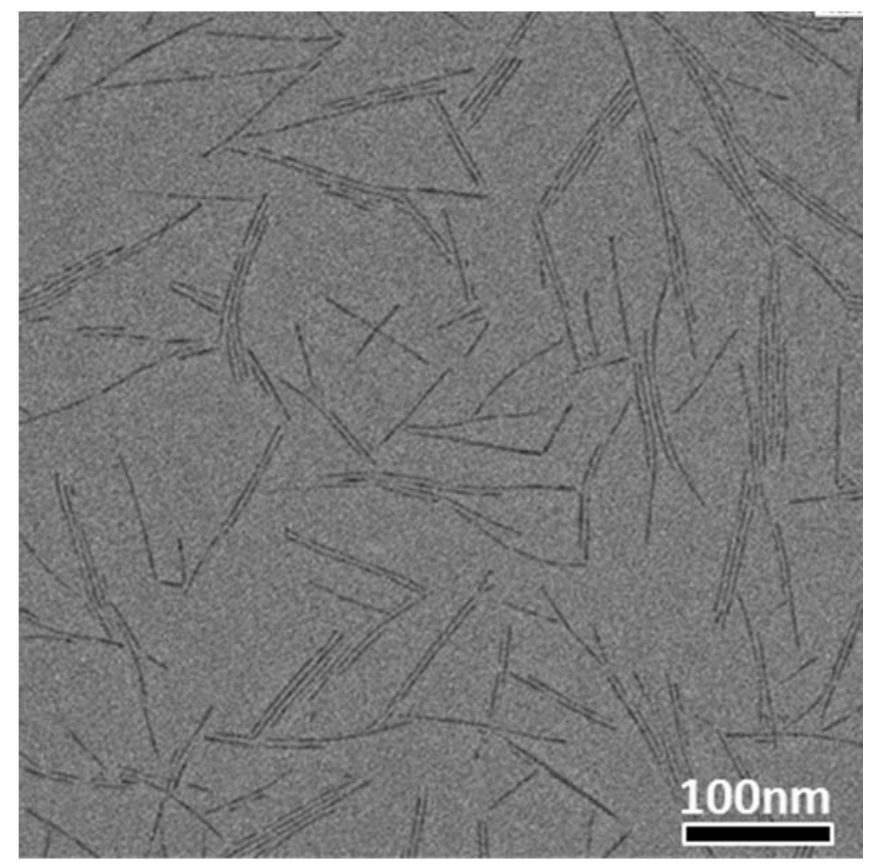

Figure S2. TEM image of wurtzite $\mathrm{ZnS}$ nanorods. 


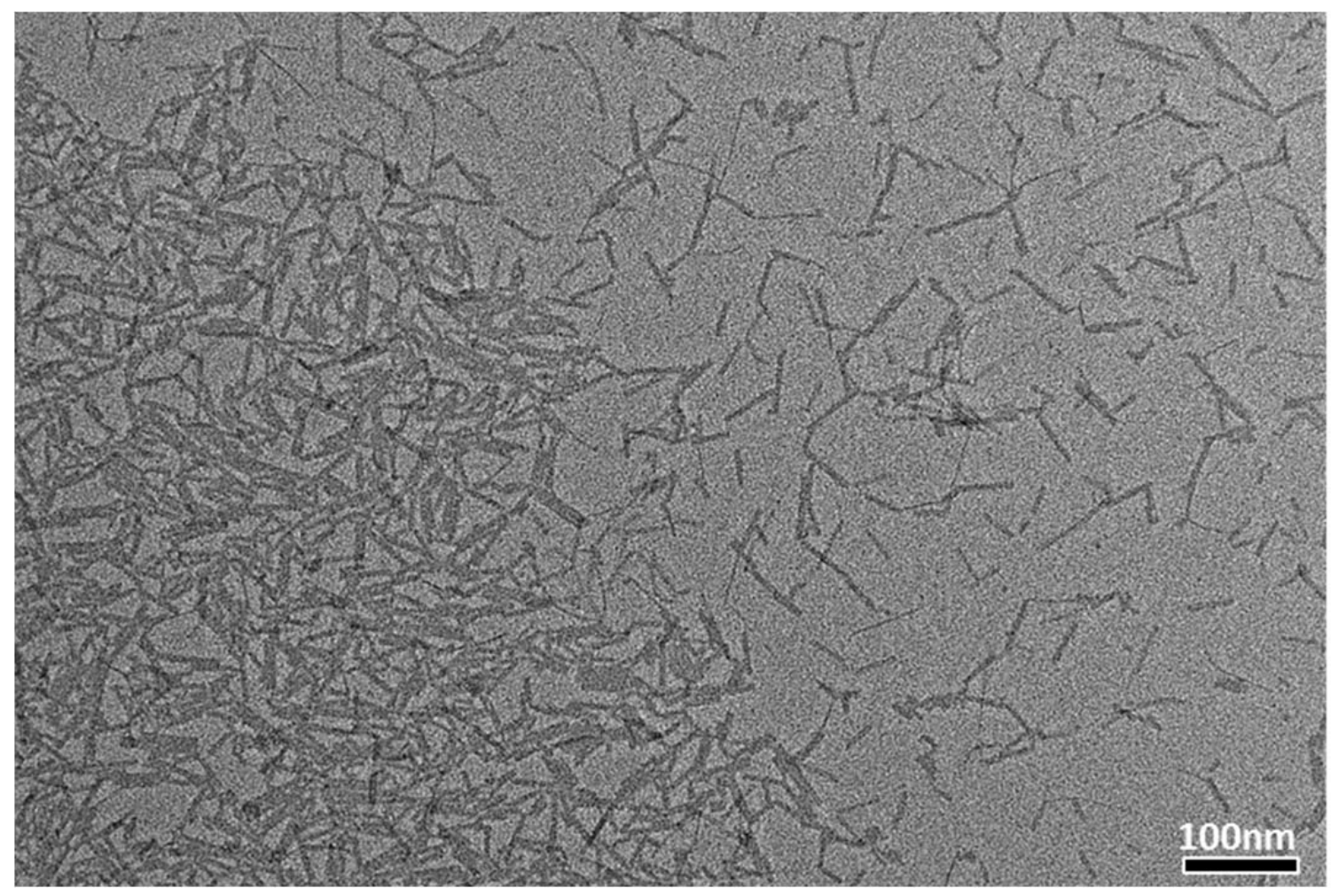

Figure S3. TEM image of wurtzite $\mathrm{ZnS}$ rectangular nanoplatelets. 

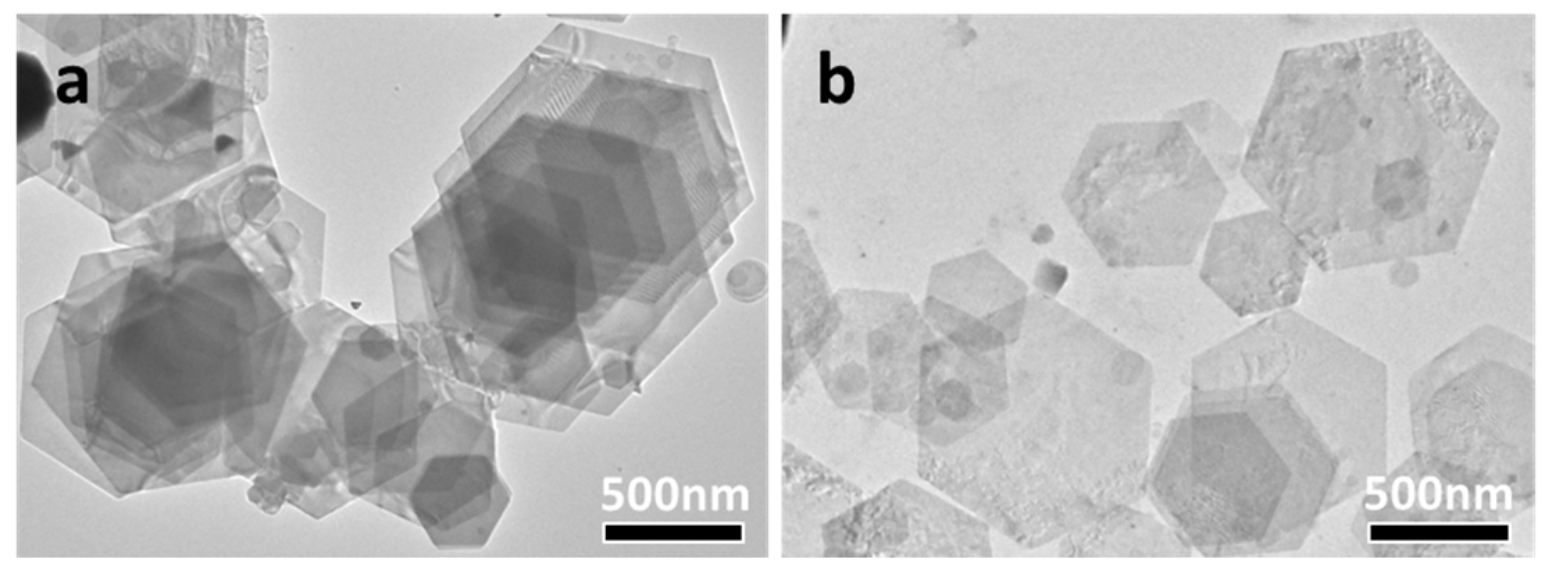

Figure S4. TEM images of a) the initial ultralarge lateral sized $\mathrm{Cu}_{2-\mathrm{x}} \mathrm{S}$ hexagonal nanoplates and b) $\mathrm{ZnS}$ hexagonal nanoplates obtained through cation exchange reactions.

Ultralarge lateral sized $\mathrm{Cu}_{2-\mathrm{x}} \mathrm{S}$ hexagonal nanoplates were prepared by Ostwald ripening method previously reported by our group. ${ }^{2}$ Although the lateral size of $\mathrm{Cu}_{2-\mathrm{x}} \mathrm{S}$ hexagonal nanoplates was increased to $1000 \mathrm{~nm}$, their thickness of $5 \mathrm{~nm}$ maintained as that of the initial samples (Figure S4). Upon cation exchange reactions, the produced $\mathrm{ZnS}$ hexagonal nanoplates retained the size and morphology as those of the initial $\mathrm{Cu}_{2-\mathrm{x}} \mathrm{S}$ hexagonal nanoplates. The percentage of the $\{002\}$ polar surfaces among all surfaces of ultralarge lateral sized $\mathrm{ZnS}$ hexagonal nanoplates was $\sim 97.8 \%$. 

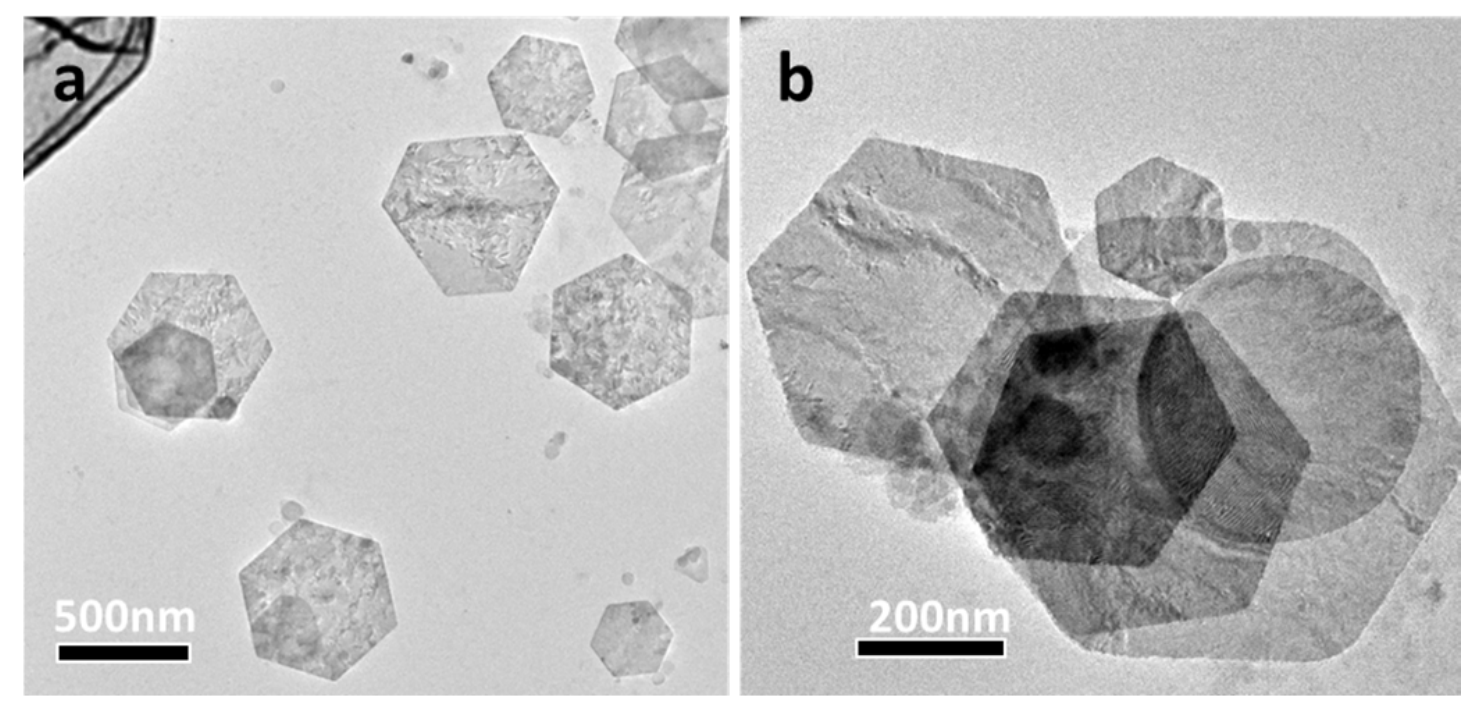

Figure S5. TEM images of ultralarge lateral sized $\mathrm{ZnS}$ nanoplates obtained through cation exchange reactions.

As depicted in Figure S5, twinkles were clearly observed in the ultralarge lateral sized ZnS hexagonal nanoplates. 


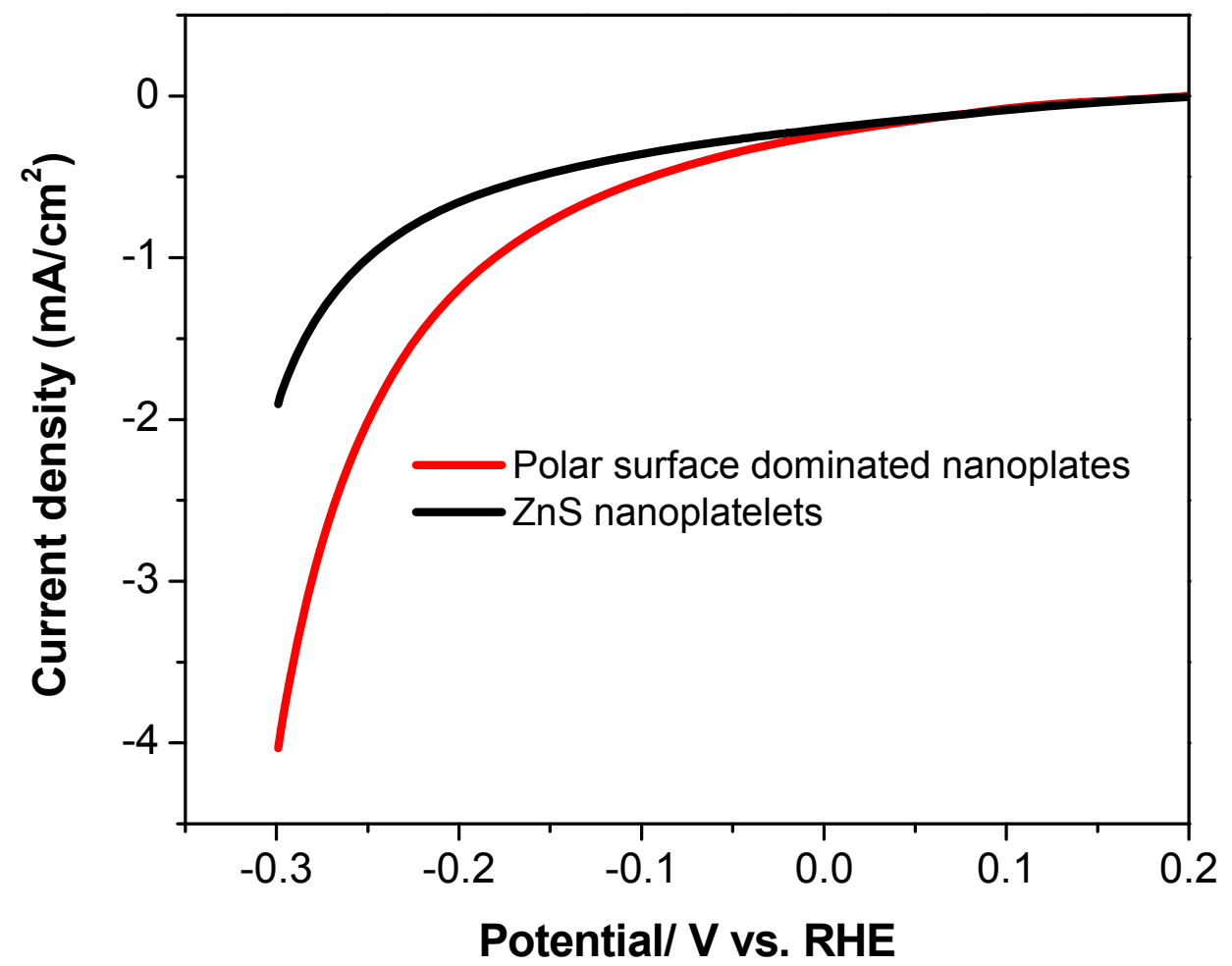

Figure S6. Comparison of HER polarization curves of polar surface dominated $\mathrm{ZnS}$ hexagonal nanoplates and $\mathrm{ZnS}$ rectangular nanoplatelets. 


\section{Surface energy calculations}
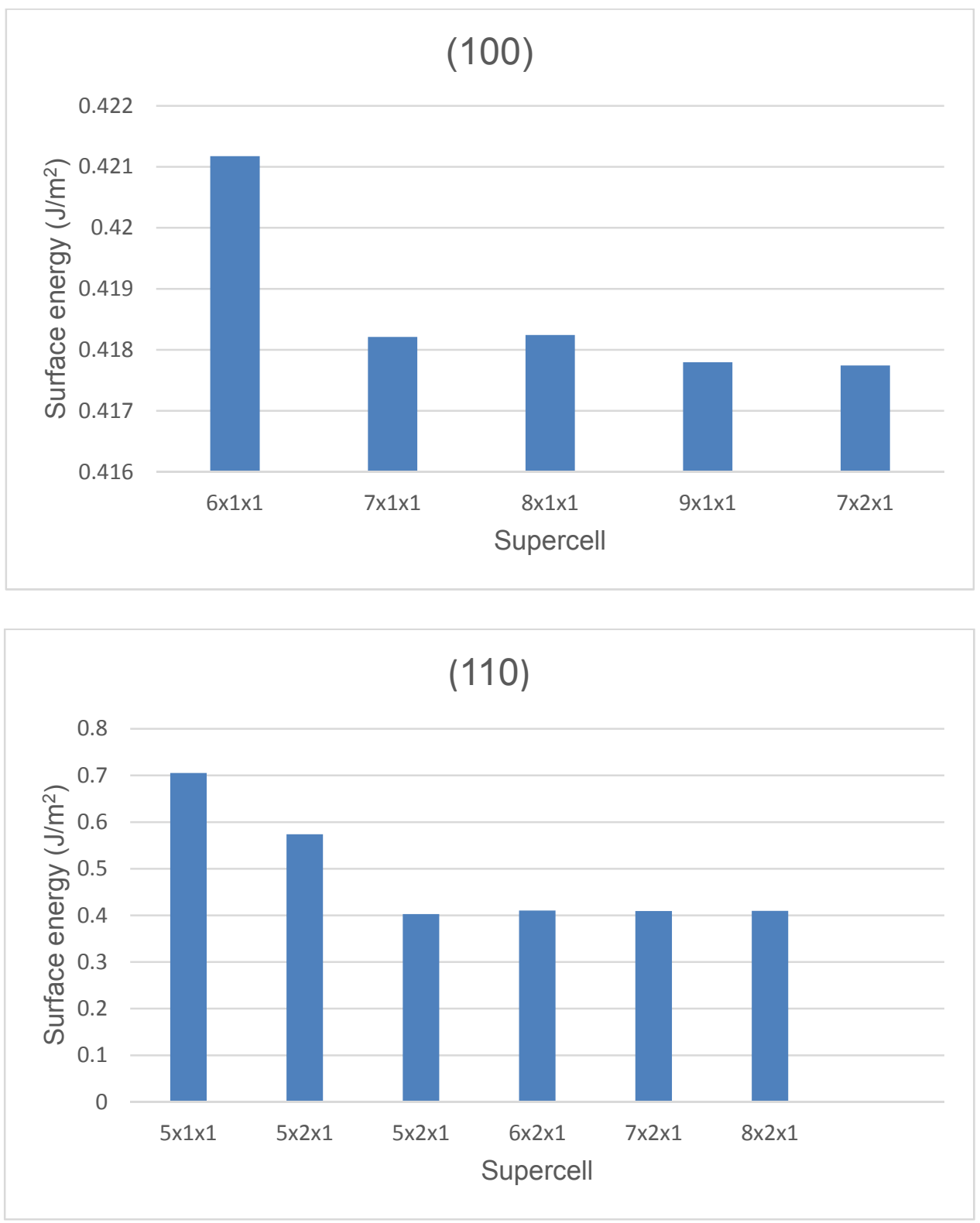

Figure S7. The convergence test of surface energy calculation for (100) and (110) surfaces. The horizontal axis shows the size of supercell by multiples of dimensions in three lattice vectors. The vertical axis shows the surface energy. 
The calculation of nonpolar surface energy is straightforward by:

$$
\gamma_{\text {surf }}=\left(E_{\text {slab }}(n \cdot \mathrm{ZnS})-E_{\text {bulk }}(n \cdot \mathrm{ZnS})\right) / 2 A
$$

where $\gamma_{\text {surf }}$ is the surface energy, $E_{\text {slab }}(n \cdot \mathrm{ZnS})$ is the energy of surface slab containing $\mathrm{n}$ formula of $\mathrm{ZnS}, E_{\text {bulk }}(n \cdot \mathrm{ZnS})$ is the energy of bulk for $\mathrm{n}$ formula of $\mathrm{ZnS}$, and $\mathrm{A}$ is the surface areas of the slab. The convergence of the surface energy has to be tested since full relaxation may be prevented by the symmetry due to small supercell and by the small number of layers. The test results are shown in Figure 1. The surface energy is $0.418 \mathrm{~J} / \mathrm{m}^{2}$ for (100) and $0.410 \mathrm{~J} / \mathrm{m}^{2}$ for (110).

In modelling, currently two techniques were used to calculate the surface energy of polar surfaces. The first is to introduce a counter charge in the vacuum region to neutralize the charge imbalance between the top and bottom layer in slab, namely dipole correction. ${ }^{16}$ This ensures a fast energy convergence with respect to the size of the supercell. The limitation of this method is that it may fail to find the lowest surface energy because of uncontrollable charge transfer between the anion-terminated and cation-terminated surface. The second technique is to hydrogenate one side of the slab to prevent dipole interaction between the top and bottom layer, ${ }^{17}$ but it only allows the study of one type of surface at once and the surface energy is not comparable to other surfaces. Based on the stabilization mechanism of polar surfaces, we develop a new method to calculate surface energy of polar surfaces, by calculating both nonpolar cation-terminated and anion-terminated surfaces in separate supercells. We establish two separate slab supercells with only cation-terminated or anion-terminated surfaces at both ends in each of them, so there is no artificial electric field in the slab supercell. We do charge transfer 
between the two supercells and calculate total energies and surface energies for each transfer. The surface energy for stoichiometric case is

$$
\gamma_{\text {surf }}=\left(E_{\text {slab }}(001) Z n+E_{\text {slab }}(001) S-E_{\text {bulk }}(n \cdot \mathrm{ZnS})\right) / 4 A
$$

and for the $\mathrm{Zn}$-terminated surface it is

$$
\gamma_{(001) Z n}=\left(E_{\text {slab }}(001) Z n-E_{\text {bulk }}(n \cdot \mathrm{ZnS})-m \cdot \mu(Z n)\right) / 2 A
$$

where $\mu(Z n)$ is the chemical potential of $\mathrm{Zn}$ and $\mathrm{m}$ is the number of excess $\mathrm{Zn}$ atoms on surface. For the S-terminated surface it is then

$$
\gamma_{(001) S}=2 \gamma_{\text {surf }}-\gamma_{(001) Z n}
$$

The method allows a full description of surface energy for all types of surface as a function of charge transfer, and we can fit out the lowest surface energy for the stoichiometric case. The method brings in some advantages: it avoids the treatment of dipole; it allows the control of charge transfer; it can determine surface energies of cation-terminated surface and anion-terminated surface separately and compare with nonpolar surfaces. 
Table S1. Calculated surface energy for stoichiometric (001) under different charge transfer (in number of electrons).

\begin{tabular}{cc}
\hline Charge transfer per formula & Surface energy \\
\cline { 2 - 2 }$(\mathrm{S}->\mathrm{Zn})$ & $\mathrm{J} / \mathrm{m}^{2}$ \\
\hline 0.0 & 2.269 \\
0.25 & 2.01 \\
0.5 & 2.175 \\
0.75 & 2.835 \\
1.0 & 3.73 \\
\hline
\end{tabular}




\section{References}

1. Pang, Y.; Uddin, M. N.; Chen, W.; Javaid, S.; Barker, E.; Li, Y.; Suvorova, A.; Saunders, M.; Yin, Z.; Jia, G. Colloidal Single-Layer Photocatalysts for MethanolStorable Solar $\mathrm{H}_{2}$ Fuel. Adv. Mater. 2019, 31, 1905540.

2. Wang, A.; Hu, X.; Wang, F.; Chen, W.; Pang, Y.; Javaid, S.; Chen, D.; Li, X.; Staaden, L.; Jia, G. Large Lateral Sized Two-Dimensional $\mathrm{Cu}_{2-\mathrm{x}} \mathrm{S}$ Nanoplates Formed by Ostwald Ripening. Mater. Lett. 2019, 237, 88-91.

3. Zhao, Y.; Zhang, Y.; Zhu, H.; Hadjipanayis, G. C.; Xiao, J. Q. Low-Temperature Synthesis of Hexagonal (Wurtzite) ZnS Nanocrystals. J. Am. Chem. Soc. 2004, 126, 6874-6875.

4. Zhang, Y.; Xu, H.; Wang, Q. Ultrathin Single Crystal ZnS Nanowires. Chem. Commun. 2010, 46, 8941-8943.

5. Jia, G.; Banin, U. A General Strategy for Synthesizing Colloidal Semiconductor Zinc Chalcogenide Quantum Rods. J. Am. Chem. Soc. 2014, 136, 11121-11127.

6. Pang, Y.; Zhang, M.; Chen, D.; Chen, W.; Wang, F.; Anwar, S. J.; Saunders, M.; Rowles, M. R.; Liu, L.; Liu, S.; Sitt, A.; Li, C.; Jia, G. Why Do Colloidal Wurtzite Semiconductor Nanoplatelets Have an Atomically Uniform Thickness of Eight Monolayers? J. Phys. Chem. Lett. 2019, 10, 3465-3471.

7. Kresse, G.; Joubert, D. From Ultrasoft Pseudopotentials to the Projector AugmentedWave Method. Phys. Rev. B 1999, 59, 1758-1775.

8. Blöchl, P. E. Projector Augmented-Wave Method. Phys. Rev. B 1994, 50, 1795317979. 
9. Kresse, G.; Furthmüller, J. Efficiency of Ab-Initio Total Energy Calculations for Metals and Semiconductors Using a Plane-Wave Basis Set. Comput. Mater. Sci. 1996, 6, $15-50$.

10. Kresse, G.; Furthmüller, J. Efficient Iterative Schemes for Ab Initio Total-Energy Calculations Using a Plane-Wave Basis Set. Phys. Rev. B 1996, 54, 11169-11186.

11. Kresse, G.; Hafner, J. Ab Initio Molecular Dynamics for Open-Shell Transition Metals. Phys. Rev. B 1993, 48, 13115-13118.

12. Perdew, J. P.; Burke, K.; Ernzerhof, M. Generalized Gradient Approximation Made Simple. Phys. Rev. Lett. 1996, 77, 3865-3868.

13. Li, Y.; Li, Y.-L.; Sa, B.; Ahuja, R. Review of Two-Dimensional Materials fFor Photocatalytic Water Splitting From a Theoretical Perspective. Catal. Sci. Tech. 2017, 7, 545-559.

14. Dudarev, S. L.; Botton, G. A.; Savrasov, S. Y.; Humphreys, C. J.; Sutton, A. P. Electron-Energy-Loss Spectra and the Structural Stability of Nickel Oxide: An LSDA+U Study. Phys. Rev. B 1998, 57, 1505-1509.

15. Miyake, T.; Zhang, P.; Cohen, M. L.; Louie, S. G. Quasiparticle Energy of Semicore $d$ Electrons in ZnS: Combined LDA + U And GW Approach. Phys. Rev. B 2006, 74, 245213(1-5).

16. Makov, G.; Payne, M. C. Periodic Boundary Conditions in Ab Initio Calculations. Phys. Rev. B 1995, 51, 4014-4022.

17. Kresse, G.; Dulub, O.; Diebold, U. Competing Stabilization Mechanism for the Polar Zno(0001)-Zn Surface. Phys. Rev. B 2003, 68, 245409(1-15). 